\title{
The effects of the genetic background on herbicide resistance fitness cost and its associated dominance in Arabidopsis thaliana
}

\author{
M Paris ${ }^{1,4,6}$, F Roux $^{1,5,6}$, A Bérard ${ }^{2,3}$ and X Reboud ${ }^{1}$ \\ ${ }^{1}$ UMR1210 Biologie et Gestion des Adventices, INRA, Dijon, France; ${ }^{2}$ Station de Génétique et Amélioration des Plantes, INRA, route de \\ St Cyr, Versailles Cedex, France and ${ }^{3}$ Centre National de Génotypage, 2 rue Gaston Crémieux, Evry Cedex, France
}

\begin{abstract}
The advantage of the resistance conferred by a mutation can sometimes be offset by a high fitness-cost penalty. This balance will affect possible fate of the resistance allele. Few studies have explored the impact of the genetic background on the expression of the resistance fitness cost and none has attempted to measure the variation in fitnesscost dominance. However, both the fitness penalty and its dominance may modify evolutionary trajectory and outcome. Here the impact of Arabidopsis thaliana intraspecific genetic diversity on fitness cost and its associated dominance was investigated by analysing 12 quantitative traits in crosses between a mutant conferring resistance to the herbicide
\end{abstract}

2,4-D and nine different natural genetic backgrounds. Fitness cost values were found to be more affected by intraspecific genetic diversity than fitness cost dominance, even though this effect depends on the quantitative trait measured. This observation has implications for the choice of the best strategy for preventing herbicide resistance development. In addition, our results pinpoint a potential compensatory improvement of the resistance fitness cost and its associated dominance by the genetic diversity locally present within a species.

Heredity (2008) 101, 499-506; doi:10.1038/hdy.2008.92; published online 3 September 2008

Keywords: auxin resistance; adaptation; fitness penalty; dominance; compensatory mutations; Arabidopsis thaliana

\section{Introduction}

Mirroring the previously observed trend for resistance to insecticides, fungicides (Holt and LeBaron, 1990) and antibiotics (Palumbi, 2001), the number of worldwide cases of herbicide resistance has increased at an exponential rate. This change has had a huge economical impact through a reduction of crops production (Heap, 2008). Herbicide resistance traits in local weed populations may originate from (1) spontaneous mutations conferring resistance in local plants, (2) migration of pollen or seeds from another population resistant to the same herbicide, or (3) introgression of transgenic herbicide resistance gene from herbicide-resistant crops and related weed species (Owen and Zelaya, 2005). In evolutionary terms, herbicide resistance could be considered as an adaptive response of a weed population to a sudden environmental change by using either new mutations (that is, spontaneous mutations) or alleles from the standing genetic variation (Orr and Betancourt,

Correspondence: Dr X Reboud, UMR1210 Biologie et Gestion des Adventices, INRA, 17 rue Sully, BP86510, Dijon 21065, France.

E-mail: reboud@dijon.inra.fr

${ }^{4}$ Current address: Génomique des Populations et Biodiversité, Laboratoire d'Ecologie Alpine, UMR CNRS-UJF 5553, Université Joseph Fourier Grenoble I, 38041 Grenoble Cedex 09, France.

${ }^{5}$ Current address: Laboratoire de Génétique et Evolution des Populations Végétales, UMR CNRS 8016, Université des Sciences et Technologies de Lille - Lille 1, F-59655 Villeneuve d'Asca cedex, France.

${ }^{6}$ These authors contributed equally to this work.

Received 30 April 2008; revised 12 June 2008; accepted 20 June 2008; published online 3 September 2008
2001). 'New mutations' means that herbicide resistance traits appear in a weed population after the first application of herbicide, whereas 'standing genetic variation' means that traits conferring herbicide resistance segregate in unexposed populations, that is, before the first application of herbicide.

According to a recent theory on adaptation, the evolutionary trajectories of 'new mutations' in a population depend on the net fitness effect associated with the adaptive allele (Orr, 1998; Barton and Keightley, 2002; also see Hastings, 2001 for a review of parasite drug resistance literature on the principal factors determining the rate at which resistance evolves). In the case of herbicide resistance, the net fitness effect results from the balance between the benefit to be resistant and the fitness cost of the resistance trait, as well as the dominances of these two variables (that is, the fitness of heterozygous RS individuals compared with resistant RR and sensitive SS homozygous individuals) (Roux et al., 2004; Roux and Reboud, 2005).

When adaptation originates from 'standing genetic variation', evolutionary trajectories of an adaptive mutation might primarily depend on its initial frequency in the population (Hermisson and Pennings, 2005). If a beneficial allele is originally neutral or weakly deleterious, its fixation probability becomes only weakly dependent on its selection coefficient after the environmental change. The reason is the high initial frequency reached by the adaptive allele before the environmental change. In weed populations, initial frequency of herbicide resistance allele in unexposed populations also depends on the fitness cost and its associated dominance (Preston and Powles, 2002). 
Although the genetic background is known to affect the fitness of the adaptive allele (Ungerer et al., 2003; Weinreich et al., 2005), and more precisely the fitness cost of herbicide resistance (Bergelson, 1994; Bergelson and Purrington, 1996), no previous experiment has been specifically designed to study the effects of the genetic background on cost dominance of herbicide resistance.

In this study, we focus on the effect of the genetic background on herbicide resistance fitness cost and its associated dominance. We used the axr1-3 mutant line, developed in the model cruciferous plant species Arabidopsis thaliana. The axr1-3 mutant line confers resistance to the 2,4-D herbicide (phenoxy-carboxylic acids group), from which different natural field resistances have been selected in weed populations (Heap, 2008). Using natural intraspecific genetic diversity in A. thaliana, we present results from an analysis of morphological and productivity-related traits in nine genetically different segregating $R / S$ populations at the $\mathrm{F}_{2}$ generation in the absence of herbicide treatment. Our main objective was to evaluate the relative sensitivity to the genetic background of the fitness cost and its associated dominance.

\section{Materials and methods}

\section{Plant materials}

The axr1-3 2,4-D resistance of the selfing species A. thaliana has been isolated from ethyl methanesulfonate (EMS) mutagenized populations of the wildtype Columbia (Col) ecotype by Estelle and Somerville (1987). The axr1-3 mutant is resistant on account of a point mutation resulting in the $\mathrm{Cys}_{154}$ to Tyr substitution (Leyser et al., 1993). In addition to 2,4-D resistance status, the axr1-3 mutant was found to affect different developmental traits, including a decrease in total seed production (Estelle and Somerville, 1987; Roux and Reboud, 2005).

To investigate the impact of $A$. thaliana intraspecific genetic diversity on the pleiotropic effects conferred by axr1-3 herbicide resistance in both the homozygous and heterozygous state, the axr1-3 resistance line was crosspollinated by hand to eight natural accessions (Bur-0, Can-0, JEA, Mt-0, Mh-1, Oy-0, PYL-1 and Sah-0). These natural accessions were chosen according to two criteria to improve the possibility to detect a genetic background effect on the axr1-3 resistance fitness cost and its associated dominance. First of all, they are present in the $A$. thaliana core-collection designed by McKhann et al. (2004). This core-collection was drawn to maximize the genetic diversity of $A$. thaliana in a reduced subset of natural accessions. Second, differences were found on a set of adaptive traits among the eight natural accessions (Reboud et al., 2004) as well as between each of the eight natural accessions and the axr1-3 resistance line (Roux F, unpublished results). The axr1-3 resistance was also crossed to the Ler genetic background, a common reference strain used in laboratory experiments. In each cross, the natural accessions and Ler strain were used as the maternal parent. Crossing success was checked by genotyping each resistance allele using an allele-specific PCR method (Roux et al., 2004). For each of the nine crosses, one resulting F1 plant was randomly chosen, then isolated and selfed to produce the F2 segregating generation with the expected resistant RR and sensitive SS homozygous individuals and RS heterozygous individuals. To limit maternal effects among crosses, all F1 plants were grown at the same time in a greenhouse, under natural light supplemented by artificial light to provide a 16-h photoperiod and temperature maintained between 20 and $25^{\circ} \mathrm{C}$. The EMS origin of the axr1-3 line means that it may carry several mutations other than the ones conferring resistance (Jander et al., 2003). In a previous study, EMS mutations other than the one conferring axr1-3 resistance were found not to induce a significant reduction of plant fitness (Roux et al., 2004). More, by our crossing protocol, any EMS mutations other than the one conferring resistance (except those closely linked to the resistance mutations) would contribute equally to the average fitness of each of the SS, RS and RR classes.

\section{Growth and quantitative traits}

To compare the relative sensitivity of the fitness cost and its associated dominance to the genetic background, an experiment involving 3120 plants was established. For each cross with a natural accession as well as with the Ler strain, 305 seeds of the F2 generation were included in the experiment. In addition, 40 Col SS seeds, 39 Ler SS seeds, 40 seeds of the axr1-3 RR line and 32 seeds of each of the eight natural accessions were added as external controls for genotyping. Seeds were sown in 30 trays $(44 \times 28.5 \mathrm{~cm})$ filled with a mix of $2 / 3$ commercial soil (Terreau Semis Bouturage Repiquage, Composana, Roche-les-Beaupré, France) and 1/3 sand, and watered two times a week without supplementary nutrients. Each tray consisted of an $8 \times 13$ array of plants. All 3120 seeds were randomized among plots and grown in the absence of herbicides in a greenhouse, under natural light supplemented by artificial light to provide a 16-h photoperiod. The temperature was maintained between 20 and $25^{\circ} \mathrm{C}$. In each tray, the 104 seeds were regularly spaced $3 \mathrm{~cm}$ apart. To keep homogeneous density throughout plant development, ungerminated seeds were replaced by extra-seedlings that were further discarded from all statistical analysis other than the calculation of segregation distortion (see Results section). The edges of trays (46 positions) were sown with seeds from 23 natural accessions (two replicates per accession) of the core-collection described by McKhann et al. (2004) to buffer against possible border effects and were discarded from the analysis. The trays were daily rotated during the growing period to avoid micro-environmental effects. The experiment stopped after senescence of all the plants.

To assess the pleiotropic fitness costs associated with the axr1-3 resistance, we extended the recorded information beyond solely seed production. Some characters (such as plant height) may still induce an ecological cost whereas they have no direct impact on seed production under optimal experimental conditions (Roux and Reboud, 2005; Wender et al., 2005). Four morphological characters were measured during the experiment: rosette diameter at the 15th and 21st day after sowing (DIAM15 and DIAM21, respectively), number of rosette leaves (LEAF) as a proxy for flowering time and height from the soil to the first flower at flowering (H1FL). The other morphological traits were measured on harvested plants: 
height from the soil to the first silique at harvest (H1SIL); maximum plant height (HMAX); the number of primary branches on the primary shoot (RAM1F); the total number of flowering heads (HEADS) measured by summing the number of flowering axes and the number of primary and secondary branches on the primary and secondary axes; and the mean distance between siliques (LEN). As measuring fitness as seed production is appropriate for a self-pollinating species (Heil and Baldwin, 2002) like $A$. thaliana, total individual fitness was assessed by the total silique length (FITNESS), a derived trait closely matching the total seed production and corresponding to the multiplication of the mean silique size (SILSIZE; average of measures on the third, fifth, seventh and ninth siliques on the primary shoot) by the total number of siliques (TOTSIL). 'Early quantitative traits' (DIAM15, DIAM21, LEAF, H1FL, H1SIL, HMAX and RAM1F) were measured for all plants from the nine crosses as well as Col SS and axr1-3 RR plants; whereas 'late quantitative traits' (HEADS, LEN, SILSIZE, TOTSIL and FITNESS) were only measured for Col SS and axr1-3 $\mathrm{RR}$ plants as well as for plants from axr1-3 $\times \mathrm{Oy}-0$ and axr1-3 $\times$ PYL-1 crosses exhibiting contrasted results for early quantitative traits.

\section{Genotyping the resistance status}

DNA was extracted from a section of the rosette leaves that was cut during the last 3 days of the experiment. Each rosette leaf section was then placed in a microcentrifuge tube containing $50 \mu \mathrm{l}$ of the extraction buffer described by Saini et al. (1999). The leaf section was crushed using a mixer mill. Tubes were placed in a water bath at $95^{\circ} \mathrm{C}$ for $6 \mathrm{~min}$, transferred onto ice for $5 \mathrm{~min}$ and vortexed for $15 \mathrm{~s}$. DNA extracts were kept at $-20^{\circ} \mathrm{C}$ before genotyping. The single nucleotide polymorphisms (SNP) conferring the axr1-3 resistance were genotyped by using the fluorescence-based Amplifluor technology (Serological Corporation, Norcross, GA, USA). This technology is based on allele-specific PCR amplification combined with the use of universal energy-transferlabeled Amplifluor primers (Giancola et al., 2006).

\section{Statistical analyses}

For each cross, the effect of axr1-3 resistance mutation on each quantitative trait in the F2 generation was assessed by a one-way analysis of variance (ANOVA). These models treat genotype (SS, RS and RR) as a fixed effect. Because the planting of F2 seeds precluded having the same genetic composition among trays (genotypes of plants are known after the end of the greenhouse experiment), a 'tray' effect was not included in the ANOVA. Although a hypothetical 'tray' effect cannot be rejected, the daily rotation of trays within the greenhouse is thought to have buffered such a 'tray' effect. Statistical analyses were performed using Systat. LEAF, RAM1F were log-transformed to meet requirements of ANOVA; whereas H1FL, HEADS, TOTSIL, SILSIZE and FITNESS were square-root transformed. The remaining variables (DIAM15, DIAM21, H1SIL and LEN) did not require transformation.

Following Roux et al. (2005b), for each 'cross $\times$ quantitative trait' combination, a distribution of cost was generated by calculating a cost value for each value of the RR dataset with their respective SS mean traits. For each cross and each quantitative trait, using these generated distributions, cost values were grouped after a Tukey pairwise comparisons test.

The dominance index was taken as:

$$
\begin{aligned}
h= & (\text { SS mean trait }- \text { RS mean trait }) /(\text { SS mean trait } \\
& - \text { RR mean trait })
\end{aligned}
$$

Following convention, the resistant allele is dominant toward cost when $h$ equals 1 , semi-dominant when $h$ equals 0.5 and recessive when $h$ approaches 0 ; overdominant and underdominant when $h$ is superior to 1 and inferior to 0 , respectively. Overdominance and underdominance indicate identical and opposite effects of RS and RR plants compared with SS plants, respectively (Roux et al., 2005b). For each 'cross $\times$ quantitative trait' combination, a distribution of dominance was generated by calculating a dominance coefficient for each value of the RS dataset with their respective SS and RR mean traits. For each cross and each quantitative trait, using these generated distributions, dominance coefficients were grouped after a Tukey pairwise comparisons test.

\section{Results}

Genetic background effect on genotypic frequencies at the F2 generation

A number of 2680 F2 plants (out of a total of 2745 F2 plants) were successfully genotyped for the axr1-3 resistance mutation. Significant segregation distortion was observed for two of the nine crosses (Table 1). For the axr1-3 $\times$ Mh- 1 and axr1-3 $\times$ Oy- 0 crosses, the RR class has fewer plants than expected and the SS class has more plants than expected. Because no reduction of survival rate was observed for the axr1-3 $\times$ Mh- 1 and axr1-3 $\times$ Oy- 0 crosses, gametes containing the axr1-3 mutation are probably associated with a lower viability and/or fertility.

\section{Herbicide resistance cost}

The effects of the genetic background on estimates of cost associated with axr1-3 resistance allele are depicted in Table 2. When comparing axr1-3 RR plants (Col background) to wild-type Col SS plants, all quantitative traits (except H1FL and HEADS) measured in this study were

Table 1 Effective class numbers and test for normal Mendelian

\begin{tabular}{|c|c|c|c|c|c|}
\hline \multirow[t]{2}{*}{ Cross } & \multicolumn{4}{|c|}{ No. of plants } & \multirow[t]{2}{*}{$\chi^{2 \mathrm{a}}$} \\
\hline & Total & $S S$ & $R S$ & $R R$ & \\
\hline axr1-3 $\times$ Bur-0 & 302 & 68 & 144 & 90 & $3.85^{\mathrm{b}}$ \\
\hline axr1-3 $\times$ Can-0 & 301 & 88 & 149 & 64 & $3.86^{\mathrm{b}}$ \\
\hline $\operatorname{axr} 1-3 \times \mathrm{JEA}$ & 293 & 79 & 151 & 63 & $2.02^{\mathrm{b}}$ \\
\hline axr1-3 × Ler & 304 & 77 & 161 & 66 & $1.86^{\mathrm{b}}$ \\
\hline $\operatorname{axr} 1-3 \times \mathrm{Mh}-1$ & 295 & 95 & 137 & 63 & $8.44^{*}$ \\
\hline $\operatorname{axr} 1-3 \times \mathrm{Mt}-0$ & 296 & 73 & 155 & 68 & $0.83^{\mathrm{b}}$ \\
\hline $\operatorname{axr} 1-3 \times \mathrm{Oy}-0$ & 298 & 96 & 141 & 61 & $9.08^{*}$ \\
\hline axr1-3 × PYL-1 & 297 & 75 & 149 & 73 & $0.03^{\mathrm{b}}$ \\
\hline $\operatorname{axr} 1-3 \times$ Sah-0 & 294 & 62 & 166 & 66 & $5.02^{\mathrm{b}}$ \\
\hline
\end{tabular}
segregation for the axr1-3 resistance allele in each of the nine crosses

${ }^{\mathrm{a}} \chi^{2}$ was calculated on the basis of expected percentages of $\frac{1}{4} \mathrm{SS}$, $\frac{1}{2}$ RS and $\frac{1}{4}$ RR with d.f.

${ }^{\mathrm{b}}$ Non-significant.

$* 0.05>P>0.01$ 
Table 2 Effect of genetic background and quantitative trait on mean estimates of cost associated with the axr1-3 herbicide resistance allele

\begin{tabular}{|c|c|c|c|c|c|c|c|c|c|c|c|c|}
\hline \multirow[t]{2}{*}{ Cross } & \multicolumn{7}{|c|}{ Early quantitative trait } & \multicolumn{5}{|c|}{ Late quantitative trait } \\
\hline & DIAM15 & DIAM21 & $L E A F$ & $H 1 F L$ & H1SIL & $H M A X$ & RAM1F & HEADS & LEN & TOTSIL & SILSIZE & FITNESS \\
\hline axr1-3 $\times$ Bur-0 & ${ }^{\mathrm{ab}} 27.2^{\mathrm{bc}}$ & ${ }^{\mathrm{ab}} 27.7^{\mathrm{c}}$ & ${ }^{\mathrm{c}} 47.9^{\mathrm{abc}}$ & - & a $20.0^{\mathrm{abc}}$ & ${ }^{\mathrm{c}} 41.2^{\mathrm{a}}$ & $\mathrm{ab} 30.0^{\mathrm{a}}$ & $\mathrm{NE}$ & NE & $\mathrm{NE}$ & $\mathrm{NE}$ & $\mathrm{NE}$ \\
\hline $\operatorname{axr} 1-3 \times$ Can -0 & ${ }^{\mathrm{a}} 21.0^{\mathrm{b}}$ & ${ }^{\mathrm{a}} 22.1^{\mathrm{abc}}$ & ${ }^{\mathrm{c}} 26.2^{\mathrm{ab}}$ & ${ }^{\mathrm{ab}} 24.2^{\mathrm{ab}}$ & ${ }^{a b} 26.3^{a b c}$ & bc $42.9^{a}$ & - & $\mathrm{NE}$ & $\mathrm{NE}$ & $\mathrm{NE}$ & $\mathrm{NE}$ & $\mathrm{NE}$ \\
\hline $\operatorname{axr} 1-3 \times \mathrm{JEA}$ & a $32.6^{c}$ & ${ }^{\mathrm{a}} 31.3^{\mathrm{c}}$ & ${ }^{\mathrm{a}} 20.1^{\mathrm{a}}$ & - & ${ }^{\mathrm{a}} 33.7^{\mathrm{bc}}$ & ${ }^{\mathrm{a}} 48.3^{\mathrm{a}}$ & - & NE & NE & $\mathrm{NE}$ & $\mathrm{NE}$ & $\mathrm{NE}$ \\
\hline axr1-3 $\times$ Ler & ${ }^{\mathrm{a}} 24.7^{\mathrm{bc}}$ & ${ }^{\mathrm{a}} 22.3^{\mathrm{ab}}$ & ${ }^{c} 51.4^{\mathrm{bc}}$ & - & ${ }^{\mathrm{a}} 19.2^{\mathrm{ab}}$ & ${ }^{\mathrm{b}} 41.3^{\mathrm{a}}$ & ${ }^{b c} 39.2^{\mathrm{ab}}$ & NE & NE & $\mathrm{NE}$ & $\mathrm{NE}$ & $\mathrm{NE}$ \\
\hline $\operatorname{axr} 1-3 \times \mathrm{Mh}-1$ & ${ }^{a b} 25.6^{b c}$ & $23.6^{\mathrm{abc}}$ & ${ }^{\mathrm{c}} 40.5^{\mathrm{abc}}$ & ${ }^{\mathrm{ab}} 13.6^{\mathrm{a}}$ & ${ }^{\mathrm{a}} 20.7^{\mathrm{abc}}$ & bc $40.4^{\mathrm{a}}$ & ${ }^{c} 45.8^{\mathrm{ab}}$ & NE & NE & $\mathrm{NE}$ & $\mathrm{NE}$ & $\mathrm{NE}$ \\
\hline $\operatorname{axr} 1-3 \times \mathrm{Mt}-0$ & a $31.4^{c}$ & ${ }^{\mathrm{a}} 33.3^{\mathrm{c}}$ & - & - & $26.4^{\mathrm{abc}}$ & ${ }^{b} 47.6^{a}$ & ${ }^{\mathrm{b}} 44.4^{\mathrm{ab}}$ & NE & NE & $\mathrm{NE}$ & NE & NE \\
\hline $\operatorname{axr} 1-3 \times \mathrm{Oy}-0$ & ${ }^{\mathrm{ab}} 25.5^{\mathrm{bc}}$ & - & ${ }^{c} 59.2^{c}$ & $\mathrm{~b} 18.5^{\mathrm{ab}}$ & ${ }^{\mathrm{a}} 13.8^{\mathrm{a}}$ & ${ }^{a b} 36.5^{a}$ & $\mathrm{~cd}_{73.3^{\mathrm{b}}}$ & ${ }^{\mathrm{cd}} 58.7$ & ${ }^{a b} 37.8^{a}$ & - & ${ }^{b c} 50.2^{b}$ & ${ }^{\mathrm{d}} 61.6^{\mathrm{ab}}$ \\
\hline axr1-3 × PYL-1 & a $24.0^{\mathrm{bc}}$ & a $25.0^{\mathrm{bc}}$ & ${ }^{a b} 29.3^{a b c}$ & ${ }^{\mathrm{ab}} 25.2^{\mathrm{ab}}$ & ${ }^{a b} 32.6^{c}$ & ${ }^{b} 42.5^{a}$ & ${ }^{c} 46.6^{\mathrm{ab}}$ & - & ${ }^{a b} 37.6^{a}$ & - & ${ }^{c} 62.9^{b}$ & ${ }^{d} 76.4^{b}$ \\
\hline $\operatorname{axr} 1-3 \times$ Sah-0 & ${ }^{\mathrm{a}} 21.4^{\mathrm{ab}}$ & ${ }^{\mathrm{a}} 22.7^{\mathrm{abc}}$ & - & ${ }^{\mathrm{b}} 43.0^{\mathrm{b}}$ & - & - & - & $\mathrm{NE}$ & NE & $\mathrm{NE}$ & $\mathrm{NE}$ & NE \\
\hline Col SS vs axr1-3 RR & ${ }^{\mathrm{a}} 10.1^{\mathrm{a}}$ & ${ }^{\mathrm{a}} 12.5^{\mathrm{a}}$ & ${ }^{\mathrm{d}} 50.3^{\mathrm{abc}}$ & - & ${ }^{\mathrm{ab}} 25.8^{\mathrm{abc}}$ & bc $38.7^{\mathrm{a}}$ & ${ }^{\mathrm{cd}} 48.3^{\mathrm{ab}}$ & - & ${ }^{b} 33.3^{a}$ & ${ }^{\mathrm{cd}} 48$ & ${ }^{\mathrm{b}} 25.5^{\mathrm{a}}$ & ${ }^{\mathrm{d}} 60.0^{\mathrm{a}}$ \\
\hline
\end{tabular}

Abbreviation: NE, not estimated.

No value ('-') indicates no difference between SS and RR plants. Different superscript letters at the right and left of the mean estimates of cost indicate a significant cross (genetic background) effect and quantitative trait effect, respectively $(P>0.05$, with Tukey correction for multiple comparisons).
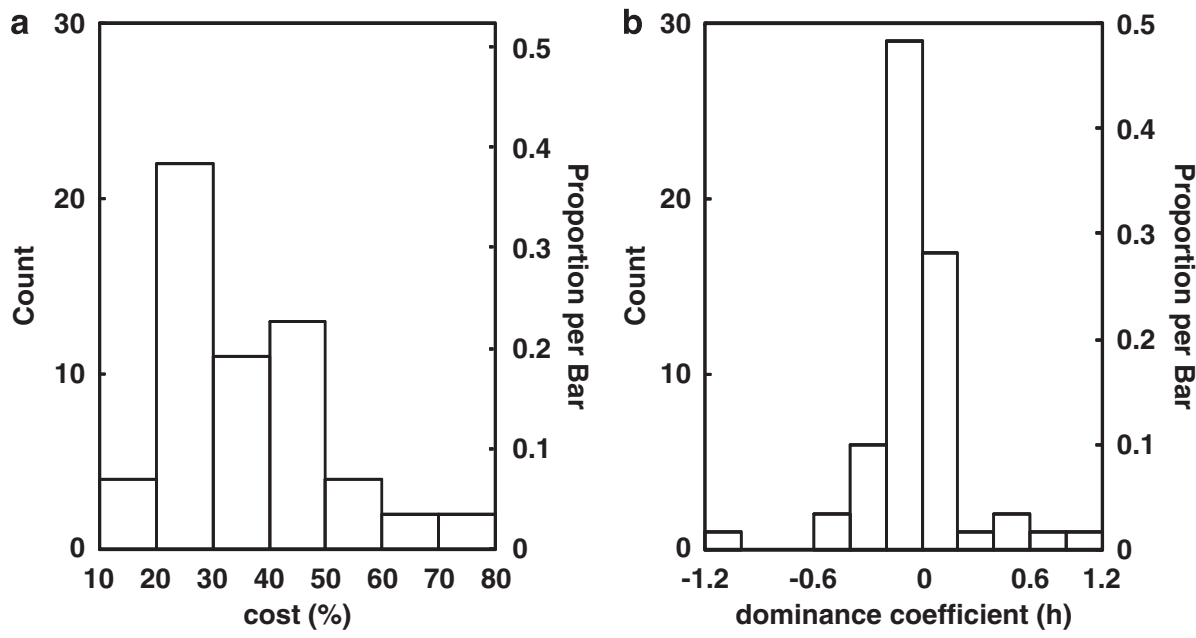

Figure 1 Overall distribution of significant cost (a) and dominance coefficient (b). The axr1-3 allele is dominant for the fitness cost when $h$ equals 1; semi-dominant when $h$ equals 0.5 ; recessive when $h$ approaches 0 ; overdominant and underdominant when $h$ is superior to 1 and inferior to 0 , respectively.

found to be affected by the axr1-3 resistance mutation. This result confirms that the axr1-3 resistance mutation affects several developmental traits, including a decrease in total seed production (Estelle and Somerville, 1987). Cost values associated with the axr1-3 resistance allele in Col genetic background are significantly different among quantitative traits (Table 2). Cost values ranged from $10.1 \%$ (reduction of rosette diameter 15 days after sowing) to $60 \%$ (reduction in fitness). The number of siliques was reduced by $48 \%$, whereas the mean silique length as a proxy of the number of seeds per silique was reduced by $25.5 \%$, leading to a global fitness reduction of $60 \%$.

Cost associated with axr1-3 resistance allele was found to depend on both cross and quantitative trait. Cost associated with the axr1-3 resistance allele was not statistically detected for $20.5 \%$ of all 'cross $\times$ quantitative trait' combinations (15 out of 73 combinations). For statistically significant costs (Figure 1a), estimates ranged from $13.6 \%$ (H1FL for axr1-3 $\times$ Mh-1 cross) to $76.4 \%$ (FITNESS for axr1-3 $\times$ PYL-1 cross), with a mean of $35.1 \%$ (s.d.=14.4\%). Because quantitative traits were not measured for all of the nine crosses, we distinguished an 'early quantitative trait' effect (nine crosses) on cost estimates from a 'late quantitative trait' effect (two crosses) on cost estimates. Over the nine crosses, 'early quantitative trait' has a significant effect on cost estimates (ANOVA, $F=8.17$, d.f. $=6, P<10^{-3}$ ) with cost estimates for LEAF, HMAX and RAM1F being higher than cost estimates for DIAM15, DIAM21, H1FL and H1SIL. No 'late quantitative trait' effect was detected (ANOVA, $F=5.31$, d.f. $=3, P=0.102$ ), certainly because of lack of statistical power (only two values for each late quantitative trait).

A significant quantitative trait effect was detected for each cross (Table 2), indicating trait-specific cost within the cross. A significant cross effect was detected for almost each quantitative trait (except HMAX and LEN; Table 2), indicating that axr1-3 resistance cost expression detected between axr1-3 RR plants and Col SS plants was modified by intra-species genetic diversity. Depending on both quantitative trait and cross, resistance cost detected between axr1-3 RR plants and Col SS plants were found to be either enhanced or reduced by genetic background. No significant cost for the total number of siliques (TOTSIL) was statistically detected in axr1- 
$3 \times$ Oy-0 and axr1-3 $\times$ PYL-1 crosses; whereas a significant cost for TOTSIL was found when comparing axr1-3 RR plants to Col SS plants. By contrast, cost for mean number of seeds per silique (SILSIZE) was at least two times enhanced in axr1-3 $\times$ Oy-0 and axr1-3 $\times$ PYL-1 crosses, in comparison to the cost detected between axr1-3 RR and Col SS plants. Total seed production (FITNESS) cost was significantly higher for axr1-3 $\times$ PYL1 crosses than the one detected between axr1-3 RR and $\mathrm{Col} S \mathrm{~S}$ plants. This result strongly suggests that both $\mathrm{Oy}-$ 0 and PYL-1 accessions may have some compensatory alleles for TOTSIL and Col accession having compensatory allele for SILSIZE. For four early quantitative traits (LEAF, H1SIL, HMAX and RAM1F), costs detected between axr1-3 RR and Col SS plants were mainly reduced in the axr $1-3 \times$ Sah- 0 cross. Sah- 0 accession is thus suggested having either several compensatory alleles, each being specific to an early quantitative trait, or one general compensatory allele common to those four early quantitative traits.

Dominance level associated with axr1-3 resistance cost The effects of genetic background on estimates of cost dominance associated with axr1-3 resistance allele are described in Table 3. For dominance coefficients $h$ (Figure 1b), estimates ranged from -1.17 (DIAM21 for axr1-3 $\times$ Oy-0 cross) to 0.81 (H1FL for axr1-3 $\times$ Oy-0 cross), with a mean closed to $0 \quad($ mean $=-0.04$, s.d. $=0.269$; without outlier ' -1.17 ': mean $=-0.02$, s.d. $=0.226$ ), indicating that axr1-3 resistance allele is almost fully recessive whatever the quantitative trait or the genetic background. Negative dominance coefficients were found for FITNESS for axr1-3 $\times$ Oy-0 and axr1-3 $\times$ PYL-1 crosses, indicating that RS plants had a higher total seed production than SS and RR plants.

For two 'cross $\times$ quantitative trait' combinations, no cost was statistically detected (DIAM21 for axr1-3 $\times$ Oy-0 cross, TOTSIL for axr1-3 $\times$ PYL-1 cross), whereas dominance coefficient $h$ was calculated, indicating that RS plants are statistically different from both SS and RR plants. For those two 'cross $\times$ quantitative trait' combinations, axr1-3 resistance allele was found highly underdominant (Table 3).

Sign epistasis, referred as the conditionality on genetic background of the sign of the phenotype effect of a mutation (Weinreich et al., 2005), was detected for cost dominance associated with axr1-3 resistance allele. For H1FL, axr1-3 resistance allele is dominant and underdominant for axr1-3 $\times$ Oy-0 and axr1-3 $\times$ Mh-1 crosses, respectively (Figure 2). The sign of axr1-3 resistance effect on dominance was also conditional on quantitative trait. For axr1-3 $\times$ JEA cross, axr1-3 resistance allele is codominant for LEAF whereas slightly underdominant for DIAM15 and DIAM21, indicating trait-specific dominance. Such a sign epistasis was not observed for cost estimates (Table 2).

Overall, dominance coefficients were less affected by genetic background and quantitative trait than cost estimates. Genetic background effect on dominance coefficients and cost estimates were found for three and six out of seven early quantitative traits, respectively. Quantitative trait effect on dominance coefficients and cost estimates was found for five and nine out of nine crosses, respectively.

We found no linear relationship between cost estimates and dominance coefficients (Pearson correlation, $n=58, r=-0.144, P=0.281)$. This result is well illustrated by H1FL (Figure 2). For axr1-3 $\times$ Mh-1 and axr1$3 \times$ Oy -0 crosses, the cost estimates were not significantly different whereas dominance coefficients were significantly different (Tables 2 and 3 ).

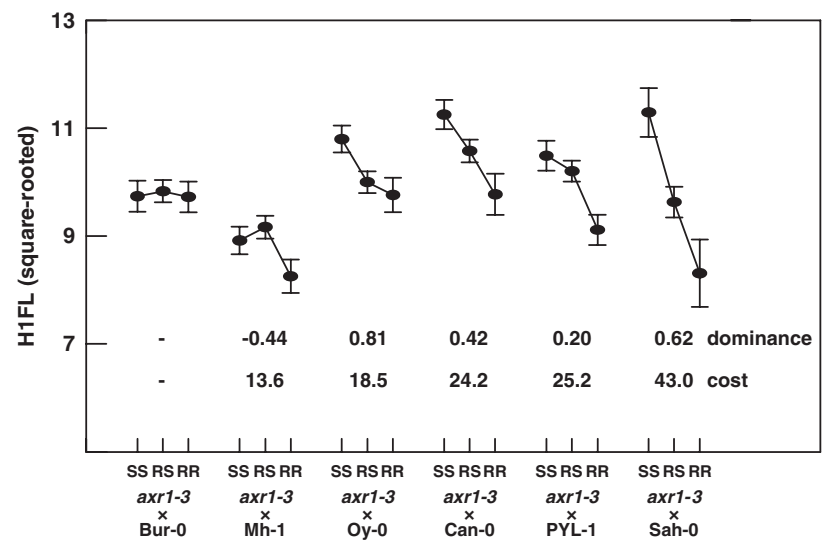

Figure 2 Effect of genetic background on estimates of cost and its associated dominance for the height to first flower at flowering (HFL). SS: AXR1-3/AXR1-3; RS: AXR1-3/axr1-3; RR: axr1-3/axr1-3. Filled circles and its associated errors bars correspond to the leastsquare mean values of the trait and its associated standard errors.

Table 3 Effect of genetic background and quantitative trait on estimates of dominance associated with the axr1-3 herbicide resistance allele

\begin{tabular}{|c|c|c|c|c|c|c|c|c|c|c|c|c|}
\hline \multirow[t]{2}{*}{ Cross } & \multicolumn{7}{|c|}{ Early quantitative trait } & \multicolumn{5}{|c|}{ Late quantitative trait } \\
\hline & DIAM15 & DIAM21 & $L E A F$ & $H 1 F L$ & H1SIL & $H M A X$ & RAM1F & HEADS & LEN & TOTSIL & SIZESIL & FITNESS \\
\hline $\operatorname{axr} 1-3 \times$ Bur-0 & ${ }^{\mathrm{a}} 0.02^{\mathrm{a}}$ & $\mathrm{a}-0.05^{\mathrm{b}}$ & ${ }^{\mathrm{a}} 0.13^{\mathrm{a}}$ & - & ${ }^{\mathrm{a}} 0.18^{\mathrm{a}}$ & ${ }^{\mathrm{a}} 0.09^{\mathrm{a}}$ & ${ }^{\mathrm{a}}-0.10^{\mathrm{a}}$ & $\mathrm{NE}$ & $\mathrm{NE}$ & $\mathrm{NE}$ & NE & $\mathrm{NE}$ \\
\hline $\operatorname{axr} 1-3 \times$ Can -0 & ${ }^{\mathrm{a}}-0.09^{\mathrm{a}}$ & ${ }^{\mathrm{a}}-0.02^{\mathrm{b}}$ & ${ }^{\mathrm{a}}-0.24^{\mathrm{a}}$ & ${ }^{\mathrm{b}} 0.42^{\mathrm{b}}$ & ${ }^{\mathrm{ab}} 0.08^{\mathrm{a}}$ & $\mathrm{ab}-0.01^{\mathrm{a}}$ & - & $\mathrm{NE}$ & NE & NE & NE & NE \\
\hline axr1-3 $\times$ JEA & $\mathrm{a}-0.15^{\mathrm{a}}$ & $\mathrm{a}-0.17^{\mathrm{b}}$ & ${ }^{b} 0.56^{b}$ & - & $\mathrm{ab}-0.04^{\mathrm{a}}$ & ${ }^{\mathrm{ab}} 0.12^{\mathrm{a}}$ & - & $\mathrm{NE}$ & NE & NE & $\mathrm{NE}$ & NE \\
\hline axr1-3 $\times$ Ler & ${ }^{\mathrm{a}} 0.04^{\mathrm{a}}$ & ${ }^{\mathrm{a}} 0.00^{\mathrm{b}}$ & ${ }^{\mathrm{a}} 0.09^{\mathrm{a}}$ & - & ${ }^{\mathrm{a}}-0.25^{\mathrm{a}}$ & ${ }^{a}-0.19^{a}$ & ${ }^{\mathrm{a}}-0.17^{\mathrm{a}}$ & NE & $\mathrm{NE}$ & $\mathrm{NE}$ & $\mathrm{NE}$ & $\mathrm{NE}$ \\
\hline $\operatorname{axr} 1-3 \times$ Mh-1 & $\mathrm{a}-0.15^{\mathrm{a}}$ & ${ }^{\mathrm{a}}-0.12^{\mathrm{b}}$ & $\mathrm{a}-0.14^{\mathrm{a}}$ & ${ }^{\mathrm{a}}-0.44^{\mathrm{a}}$ & ${ }^{\mathrm{a}}-0.10^{\mathrm{a}}$ & ${ }^{\mathrm{a}} 0.13^{\mathrm{a}}$ & $\mathrm{a}-0.06^{\mathrm{a}}$ & $\mathrm{NE}$ & $\mathrm{NE}$ & $\mathrm{NE}$ & NE & NE \\
\hline $\operatorname{axr} 1-3 \times \mathrm{Mt}-0$ & ${ }^{\mathrm{a}}-0.01^{\mathrm{a}}$ & ${ }^{\mathrm{a}}-0.01^{\mathrm{b}}$ & - & - & ${ }^{\mathrm{a}} 0.07^{\mathrm{a}}$ & ${ }^{\mathrm{a}} 0.12^{\mathrm{a}}$ & ${ }^{\mathrm{a}}-0.02^{\mathrm{a}}$ & NE & NE & $\mathrm{NE}$ & NE & NE \\
\hline axr1-3 $\times$ Oy-0 & ${ }^{\mathrm{bc}} 0.00^{\mathrm{a}}$ & $\mathrm{a}-1.17^{\mathrm{a}}$ & ${ }^{\mathrm{bc}} 0.08^{\mathrm{a}}$ & ${ }^{c} 0.81^{b}$ & bc $-0.10^{\mathrm{a}}$ & $\mathrm{b}-0.06^{\mathrm{a}}$ & ${ }^{\mathrm{bc}} 0.06^{\mathrm{a}}$ & $a b c-0.06$ & $a b c-0.14^{a}$ & - & $a b c-0.35^{a}$ & $a b c-0.39^{a}$ \\
\hline axr1-3 × PYL-1 & $\mathrm{ab}-0.14^{\mathrm{a}}$ & $\mathrm{ab}-0.27^{\mathrm{b}}$ & ${ }^{\mathrm{ab}} 0.09^{\mathrm{a}}$ & ${ }^{\mathrm{b}} 0.20^{\mathrm{ab}}$ & $\mathrm{ab}^{\mathrm{b}}-0.13^{\mathrm{a}}$ & $\mathrm{ab}-0.13^{\mathrm{a}}$ & ${ }^{\mathrm{ab}} 0.08^{\mathrm{a}}$ & - & $\mathrm{ab}-0.10^{\mathrm{a}}$ & a -0.49 & ${ }^{a b}-0.03^{a}$ & ${ }^{a b}-0.25^{a}$ \\
\hline $\operatorname{axr} 1-3 \times$ Sah-0 & ${ }^{\mathrm{a}}-0.01^{\mathrm{a}}$ & ${ }^{\mathrm{a}}-0.01^{\mathrm{b}}$ & - & ${ }^{\mathrm{b}} 0.62^{\mathrm{b}}$ & - & - & - & $\mathrm{NE}$ & $\mathrm{NE}$ & NE & NE & NE \\
\hline
\end{tabular}

Abbreviation: NE, not estimated.

No value ('-') indicates no difference among SS, RS and RR plants. Different superscript letters at the right and left of the mean estimates of dominance indicate a significant cross (genetic background) effect and quantitative trait effect, respectively $(P>0.05$, with Tukey correction for multiple comparisons). 


\section{Discussion}

\section{Genetic background may affect herbicide resistance dynamics}

The fitness effect of a mutation, and its dominance determine the expected evolutionary trajectories of the corresponding trait. Previous empirical estimates have primarily been based on the observation of evolutionary equilibrium of resistance genes in natural populations (Purrington, 2000). Most models studying the dynamics of resistance in plant populations therefore incorporate the fitness costs associated with resistance (herbivores: Restif and Koella, 2004; pathogens: Stahl et al., 1999; Roy and Kirchner, 2000; Burdon and Thrall, 2003; herbicide: Neve et al., 2003; Roux et al., 2008). Most models have considered the resistance trait to be constant. However, by analyzing the same resistance mutation in eight different segregating genetic backgrounds, our results strongly suggest that the fitness cost of a resistant genotype and its associated dominance should rather be considered variable in natural populations, depending on local genetic composition. The effects of genetic background on cost of herbicide resistance have already been described in weed species (for a review, see Bergelson and Purrington, 1996). To our knowledge, no previous experiment was specifically designed to study the effects of genetic background on either distortion of segregation or cost dominance of herbicide resistance. Because distortion of segregation disadvantaged the axr1-3 resistance allele in two genetic backgrounds, cost estimates and dominance coefficients are biased downward for axr1-3 $\times$ Mh- 1 and axr1-3 $\times 0 \mathrm{y}-0$ crosses. It is therefore important to include the analysis of meiotic drive when estimating cost and dominance (both at the individual level and the population level).

We have shown that the dominance coefficient is less sensitive to genetic background than the cost. The mean dominance coefficient associated with the axr1-3 resistance allele was close to that previously found $(h=0.07)$ in an experiment investigating epistatic interactions among three herbicide resistance alleles in A. thaliana (including axr1-3 resistance allele; Roux et al., 2005b). This low value is consistent with Wright's physiological theory of dominance (Wright, 1977), which proposes that enzymes activity is seldom limiting, because of excess activity of wild-type enzymes (Kacser and Burns, 1981). This type of explanation would be consistent with an absence of relationship between cost estimates and dominance coefficients. Nevertheless some genetic background effects on dominance were detected for several quantitative traits, suggesting that the 'safety margin' associated with the AXR1 gene (a value describing the maximum decrease of the enzyme activity that can be tolerated without affecting the phenotype; Wright, 1934) might be trait and/or development dependent.

Because most spontaneous mutations initially appear in the heterozygous state, the initial dynamics in the absence of herbicide will depend on both the fitness cost and its dominance. However, the results suggest a relatively small effect of genetic background on the dominance; consequently, the main difference between populations in mutation-drift equilibrium will depend on the expressed within-population fitness cost.
Because the evolutionary trajectories of an adaptation originating from standing genetic variation depend on its initial frequency (Hermisson and Pennings, 2005), the initial allele frequency in population before the first herbicide treatment is a crucial parameter. This value is highly dependent on the genetic background, and so it is highly unpredictable. To date, only one study has estimated the initial frequency of the herbicide resistance allele in a one weed population of Lolium rigidum (Preston and Powles, 2002). Further experiments estimating initial resistance allele frequencies in different weed populations would be of great value to consider appropriate management strategies (Roux et al., 2008).

\section{Ecological implications of trait-specific cost and dominance}

In natural conditions, the outcome of selection might depend on trait-specific cost and dominance (van Dooren, 2006). In environments with no competition, one can expect genetic backgrounds conferring a cost for early traits but no cost for seed production to be selected for, whereas those conferring the converse (no cost for early traits but a cost for seed production) would be selected against. In competitive environments, the opposite result might well be expected as competition will tend to favour genotypes with the highest early vigour. For example, plants without cost on seed production but with reduced rosette diameter may strongly suffer of competition compared with plants without reduced rosette diameter but with cost on seed production. This pattern has been observed for the csr1-1 herbicide resistance allele in experimental settings with $A$. thaliana in greenhouse experiments (Roux et al., 2005a, 2006).

Because genetic variation for trait-specific cost and dominance was observed in our study for axr1-3 resistance allele, the timing at which the cost and its associated dominance are expressed might also be selected. In the same way $A$. thaliana lines associated with a high seed production in the fall because of adequate germination timing can be counter-selected in spring on account of a delay in germination timing (Donohue, 2002; Donohue et al., 2005). As a result, the dynamics of selection of an adaptive allele might, in turn, depend on the interactions between environment and timing of trait expression.

\section{Compensatory evolution of herbicide resistance cost}

Two modes of ameliorative evolution have been suggested to reduce the pleiotropic fitness costs. First, an adaptive mutation conferring a fitness cost could be replaced by another mutation conferring the same adaptation but with a lower cost (Cohan et al., 1994). This 'replacement' mode of amelioration may occur at a single gene (allelic replacement; Guillemaud et al., 1998) or at different genes (nonallelic replacement; Lenski, 1988; Cohan et al., 1994). Second and non-exclusively, the pleiotropic fitness costs may be shaped by natural selection of modifiers at other loci. That is, selection can occur at genes whose products interact with the product of the adaptive allele (Fisher, 1928). This 'compensatory' mode of amelioration could occur either by the accumulation of compensatory mutations in the genetic background containing the original adaptive mutation (Andersson, 2003) or by the contribution of 
the genetic diversity already present within the local population of the corresponding species (Cohan et al., 1994). Factors affecting the accumulation of compensatory mutations have been extensively studied in organisms with a rapid life cycle: bacteria, yeast and fungi. These studies identified factors including the rate of compensatory mutation (Poon and Chao, 2005; Gagneux et al., 2006; Schoustra et al., 2006), the environment (Björkman et al., 2000; Reynolds, 2000) and the size of a population bottleneck (Levin et al., 2000; Maisnier-Patin et al., 2002). In organisms with longer generation time like weed species (often, only one generation per year), it is tempting to speculate that awaiting compensatory mutations might be less efficient than making use of the genetic diversity already present in the species. Although the accumulation of compensatory mutations in the genetic background containing the resistance allele seems hard to test in plant species, it is possible to survey intra-species genetic diversity to estimate the number of compensatory genes and their relative effects. Our results suggest that different compensatory genes are segregating in different genetic backgrounds.

\section{Acknowledgements}

We thank Mathilde Dufaÿ specially for her helpful discussion and comments on an earlier version of this paper. We are grateful to Annick Matéjicek, Guillaume Bay, Séverine Michel, Bertrand Jacquemin and greenhouse staff from the SEDE Experimental Unit for their technical assistance. This study was supported by a grant to FR from Bayer Crop Sciences. High-throughput genotyping has received funding from AIP INRA 'Séquençage Végétal et Post-séquençage' as well as INRA 'Santé des Plantes and Environnement' projects.

\section{References}

Andersson DI (2003). Persistence of antibiotic resistant bacteria. Curr Opin Microbiol 6: 452-456.

Barton NH, Keightley PD (2002). Understanding quantitative genetic variation. Nat Rev Genet 3: 11-21.

Bergelson J (1994). The effects of genotype and the environment on costs of resistance in lettuce. Am Nat 143: 349-359.

Bergelson J, Purrington CB (1996). Surveying patterns in the cost of resistance in plants. Am Nat 148: 536-558.

Björkman J, Nagaev I, Berg OG, Highes D, Andersson DI (2000). Effects of environment on compensatory mutations to ameliorate costs of antibiotic resistance. Science 287: 1479-1482.

Burdon JJ, Thrall PH (2003). The fitness costs to plants of resistance to pathogens. Genome Biol 4: 227.1-227.3.

Cohan FM, King EC, Zawadzki P (1994). Amelioration of the deleterious pleiotropic effects of an adaptive mutation in Bacillus subtilis. Evolution 48: 81-95.

Donohue K (2002). Germination timing influences natural selection on life-history characters in Arabidopsis thaliana. Ecology 83: 1006-1016.

Donohue K, Dorn L, Griffith C, Kim E, Aguilera A, Polisetty CR et al. (2005). Niche construction through germination cueing: life-history responses to timing of germination in Arabidopsis thaliana. Evolution 59: 771-785.

Estelle MA, Somerville C (1987). Auxin-resistant mutants of Arabidopsis with an altered morphology. Mol Gen Genet 206: 200-206.

Fisher RA (1928). The possible modification of the responses of wild type to recurrent mutations. Am Nat 62: 115-126.
Gagneux S, Davis Long C, Small PM, Van T, Schoolnik GK, Bohannan BJM (2006). The competitive cost of antibiotic resistance in Mycobacterium tubercolusis. Science 312: 1944-1946.

Giancola S, McKhann HI, Bérard A, Camilleri C, Durand S, Libeau P et al. (2006). Utilization of the three high-throughput SNP genotyping methods, the GOOD assay, Amplifluor and TaqMan, in diploid and polyploid plants. Theor Appl Genet 112: 1115-1124.

Guillemaud T, Lenormand T, Bourguet D, Chevillon C, Pasteur N, Raymond M (1998). Evolution of resistance in Culex pipiens: allele replacement and changing environment. Evolution 52: 443-453.

Hastings IM (2001). Modelling parasite drug resistance: lessons for management and control strategies. Tropical Med Int Health 6: 883-890.

Heap I (2008). International survey of herbicide resistant weeds. [WWW document]. http://www.weedscience.com.

Heil M, Baldwin T (2002). Fitness costs of induced resistance: emerging experimental support for a slippery concept. Trends Plant Sci 7: 61-67.

Hermisson J, Pennings PS (2005). Soft sweeps: molecular population genetics of adaptation from standing genetic variation. Genetics 169: 2335-2352.

Holt JS, LeBaron HM (1990). Significance and distribution of herbicide resistance. Weed Technol 4: 141-149.

Jander G, Baerson SR, Hudak JA, Gonzalez KA, Gruys KJ, Last RL (2003). Ethylmethanesulfonate saturation mutagenesis in Arabidopsis to determine frequency of herbicide resistance. Plant Physiol 131: 139-146.

Kacser H, Burns JA (1981). The molecular basis of dominance. Genetics 97: 639-666.

Lenski RE (1988). Experimental studies of pleiotropy and epistasis in Escherichia coli. II. Compensation for maladaptive effects associated with resistance to virus T4. Evolution 42: 433-440.

Levin BR, Perrot V, Walker N (2000). Compensatory mutations, antibiotic resistance and the population genetics of adaptive evolution in bacteria. Genetics 154: 985-997.

Leyser HMO, Lincoln CA, Timpte C, Lammer D, Turner J, Estelle M (1993). Arabidoposis auxin-resistance gene AXR1 encodes a protein related to ubiquitin-activating enzyme E1 Nature 364: 161-164.

Maisnier-Patin S, Berg OG, Liljas L, Andersson DI (2002). Compensatory adaptation to the deleterious effect of antibiotic resistance in Salmonella typhimurium. Mol Microbiol 46: 355-366.

McKhann HI, Camilleri C, Bérard A, Bataillon T, David JL, Reboud X et al. (2004). Nested core collections maximizing genetic diversity in Arabidopsis thaliana. Plant J 38: 193-202.

Neve P, Diggle AJ, Smith FP, Powles SB (2003). Simulating evolution of glyphosate resistance in Lolium rigidum II: past, present and future glyphosate use in Australian cropping. Weed Res 43: 418-427.

Orr HA (1998). The population genetics of adaptation: the distribution of factors fixed during adaptive evolution. Evolution 52: 935-949.

Orr HA, Betancourt AJ (2001). Haldane's sieve and adaptation from standing genetic variation. Genetics 157: 875-884.

Owen MDK, Zelaya IA (2005). Herbicide-resistant crops and weed resistance to herbicides. Pest Manag Sci 61: 301-311.

Palumbi SR (2001). Humans as the world's greatest evolutionary force. Science 293: 1786-1790.

Poon AFY, Chao L (2005). The rate of compensatory mutation in the DNA bacteriophage $\phi 174$. Genetics 170: 989-999.

Preston C, Powles SB (2002). Evolution of herbicide resistance in weeds: initial frequency of target site-based resistance to acetolactate synthase-inhibiting herbicides in Lolium rigidum. Heredity 88: 8-13.

Purrington CB (2000). Costs of resistance. Curr Opin Plant Biol 3 : 305-308. 
Reboud X, Le Corre V, Scarcelli N, Roux F, David JL, Bataillon T et al. (2004). Natural variation among accessions of Arabidopsis thaliana: beyond the flowering date, what morphological traits are relevant to study adaptation? In: Cronk QC, Whitton J, Taylor IEP (eds). Plant Adaptation: Molecular Biology and Ecology. NRC Research Press: Ottawa, Canada. pp 135-142.

Restif O, Koella JC (2004). Concurrent evolution of resistance to tolerance to pathogens. Am Nat 164: E90-E102.

Reynolds MG (2000). Compensatory evolution in rifampicinresistant Escherichia coli. Genetics 156: 1471-1481.

Roux F, Camilleri C, Bérard A, Reboux X (2005a). Multigenerational versus single generation studies to estimate herbicide resistance fitness cost in Arabidopsis thaliana. Evolution 59: 2264-2269.

Roux F, Camilleri C, Giancola S, Brunel D, Reboud X (2005b). Epistatic interactions among herbicide resistances in Arabidopsis thaliana: the fitness cost of multiresistance. Genetics 171: 1277-1288.

Roux F, Gasquez J, Reboud X (2004). The dominance of the herbicide resistance cost in several Arabidopsis thaliana mutant lines. Genetics 166: 449-460.

Roux F, Giancola S, Durand S, Reboud X (2006). Building of an experimental cline with Arabidopsis thaliana to estimate herbicide fitness cost. Genetics 173: 1023-1031.

Roux F, Paris M, Reboud X (2008). Delaying weed adaptation to herbicide by environmental heterogeneity: a simulation approach. Pest Manag Sci 64: 16-29.

Roux F, Reboud X (2005). Is the cost of herbicide resistance expressed in the breakdown of the relationships between characters? A case study using synthetic auxin resistant Arabidopsis thaliana mutants. Genet Res 85: 101-110.
Roy BA, Kirchner JW (2000). Evolutionary dynamics of pathogen resistance and tolerance. Evolution 54: 51-63.

Saini HS, Shepherd M, Henry RJ (1999). Microwave extraction of total genomic DNA from barley grains for use in PCR. J I Brewing 105: 185-190.

Schoustra SE, Debets AJM, Slakhorst M, Hoekstra RF (2006). Reducing the cost of resistance; experimental evolution in the filamentous fungus Aspergillus nidulans. J Evolution Biol 19: $1115-1127$.

Stahl EA, Dwyer G, Mauricio R, Kreitman M, Bergelson J (1999). Dynamics of disease resistance polymorphism at the Rpm1 locus in Arabidopsis. Nature 400: 667-671.

Ungerer MC, Linder CR, Rieseberg LH (2003). Effects of genetic background on response to selection in experimental populations of Arabidopsis thaliana. Genetics 163: 277-286.

van Dooren TJM (2006). Protected polymorphism and evolutionary stability in pleiotropic models with trait-specific dominance. Evolution 60: 1991-2003.

Weinreich DM, Watson RA, Chao L (2005). Perspective: sign epistasis and genetic constraint on evolutionary trajectories. Evolution 59: 1165-1174.

Wender NJ, Polisetty CR, Donohue K (2005). Density-dependent processes influencing the evolutionary dynamics of dispersal: a functional analysis of seed dispersal in Arabidopsis thaliana (Brassicaceae). Am J Bot 92: 960-971.

Wright S (1934). Physiological and evolutionary theories of dominance. Am Nat 68: 25-53.

Wright $S$ (1977). The evolution of dominance. In: Wright $S$ (ed). Evolution and the Genetics of Populations. Volume 3: Experimental Results and Evolutionary Deductions. The University of Chicago Press: Chicago. pp 498-526. 